\title{
On Groups Whose Irreducible Character Degrees of All Proper Subgroups are All Prime Powers
}

\author{
Shitian Liu \\ School of Mathematics, Sichuan University of Arts and Science, Dazhou, Sichuan, 635000, China \\ Correspondence should be addressed to Shitian Liu; s.t.liu@yandex.com \\ Received 13 May 2021; Accepted 2 June 2021; Published 16 June 2021 \\ Academic Editor: Jie $\mathrm{Wu}$ \\ Copyright (C) 2021 Shitian Liu. This is an open access article distributed under the Creative Commons Attribution License, which \\ permits unrestricted use, distribution, and reproduction in any medium, provided the original work is properly cited. \\ Isaacs, Passman, and Manz have determined the structure of finite groups whose each degree of the irreducible characters is a \\ prime power. In particular, if $G$ is a nonsolvable group and every character degree of a group $G$ is a prime power, then $G$ is \\ isomorphic to $S \times A$, where $S \in\left\{A_{5}, \mathrm{PSL}_{2}(8)\right\}$ and $A$ is abelian. In this paper, we change the condition, each character degree of a \\ group $G$ is a prime power, into the condition, each character degree of the proper subgroups of a group is a prime power, and give \\ the structure of almost simple groups whose character degrees of all proper subgroups are all prime powers.
}

\section{Introduction}

In this paper, we assume that all groups are finite. Denote by $\operatorname{Irr}(G)$ the set of all complex irreducible characters of a group $G$, write the set of linear characters of $G$ by $\operatorname{Lin}(G)$, and denote the set of character degrees of a group $G$ by $\operatorname{cd}(G)$.

Let $G$ be a group and let $p, q$ be different primes. If $\operatorname{cd}(G)=\{1, m\}$, then either $G$ has an abelian normal subgroup of index $m$ or $m=p^{e}$ for a prime $p$ and $G$ is the direct product of a $p$-group and an abelian group (see Theorem 12.5 in [1]); Isaacs and Passman have identified the structure of groups with $\operatorname{cd}(G) \subseteq\{1, p, q\}$ and $\operatorname{cd}(G) \subseteq\left\{1, p, p^{2}\right\}$ (see $[2,3]$, respectively).

In this paper, the following problem is considered.

Question: what can we say about the structure of a group if irreducible character degrees of all proper subgroups are all of prime powers.

In order to argue in short, we introduce the following definition.

Definition 1. An irreducible character $\chi \in \operatorname{Irr}(G)$ is a $D P$-character if $\chi(1)$ is a prime power, and a non$D P$-character otherwise. A group $G$ is named a DP-group if, for every $\chi \in \operatorname{Irr}(G), \chi$ is a DP-character, and a non-
DP-group otherwise. We also assume that an abelian group is a DP-group.

Manz in $[4,5]$ has determined the structure of DP-groups. In particular, nonsolvable DP-groups are determined.

Theorem 1 (see [5]). If $G$ is a nonsolvable DP-group, then $G=S \times A$, where $A$ is abelian and $S$ is isomorphic to $A_{5}$ or $\mathrm{PSL}_{2}(8)$.

In order to talk conveniently, we give a definition.

Definition 2. Let $\sum G$ be the set of all proper subgroups of a group $G$. A group $G$ is called an SDP-group if, for each $M \in \sum G, \quad M$ is a DP-group, and a non-SDP-group otherwise.

We will prove the following results.

Theorem 2. Let $G$ be an almost simple group such that $S \leq G \leq A u t(S)$, where $S$ is a nonabelian simple group. Assume that $G$ is an SDP-group, then $G$ is isomorphic to one of the groups: $P S L_{2}(q)$, where $q=2 r^{m}+1 \geq 5$ is a prime with $r$ an odd prime or $q=2^{m}$ and $2^{m}-1 \geq 3$ is a prime, $\operatorname{PSL}_{2}(9)$, $\mathrm{PSL}_{2}\left(3^{5}\right), \mathrm{S}_{5}$, or $\mathrm{PSL}_{3}(3)$.

We will introduce the structure of this paper. In Section 2 , we give the structure of simple SDP-groups and some 
properties of SDP-groups; in Section 3, we give the structure of almost simple SDP-groups. For a group $G$, let $\sum G$ be the set of all proper subgroups of $G$ and $\max G$ be the set of representatives of maximal subgroups of $G$. The notation and notions are standard, see $[1,6]$, for instance.

\section{Some Lemmas}

In this section, we will give some needed results that are used to prove main theorem. We first give some basic results of SDP-group, then some subgroup of the nonabelian simple groups is given, and finally the structure of the nonabelian simple SDP-groups is determined. Now, we give some results about SDP-groups.

\section{Lemma 1}

(1) $G$ is an SDP-group if and only if, for each $M \in \sum G$, $M$ is a DP-group.

(2) Let $G$ be an SDP-group and let $N$ be a nontrivial normal subgroup of $G$. Then, $N$ and $G / N$ are DP-groups.

(3) A DP-group must be an SDP-group.

Proof

(1) We can get the result by Definition 2 .

(2) $N$ is a DP-group by Definition 2 , and $1<N<G$. If $G / N$ is not a DP-group, then there exists a subgroup $M \in \max G$ with $N \not M M$, so $G=M N$ and $1<M<G$, $M$ is a DP-group. Since $(G / N) \cong(M / M \cap N)$ is not a DP-group, there is a character $\chi \in \operatorname{Irr}(M / N \cap M)$ which is not a DP-character. It follows that $M$ is not a DP-group, a contradiction.

(3) Let $G$ be a DP-group. We will show that $G$ is an SDP-group. If $G$ is not an SDP-group, then there is a subgroup $M \in \sum G$ and an irreducible character $\chi \in \operatorname{Irr}(M)$ such that $\chi$ is a non-DP-character. Let $I=I_{G}(\chi)$, the inertia group of $\chi$ in $G$. Let $\eta \in \operatorname{Irr}(I)$ such that $\left[\eta_{M}, \chi\right] \neq 0$. Then, by Theorem 6.5 of [1], $\eta^{G} \in \operatorname{Irr}(G)$. We know that $\chi(1)|\eta(1)| \eta^{G}(1)$, so there is a maximal subgroup $K \in \sum G$ and an irreducible character $\xi \in \operatorname{Irr}(K)$ such that $\xi$ is not a DP-character, so $K$ is not a DP-group. It follows that $G$ is not a DP-group, a contradiction.

In generality, a group, which is the direct product of two DP-groups, is not an SDP-group.

Example 1. Let $P, Q$ be nonabelian $p$ - and $q$-groups for different primes $p, q$, respectively. Also, assume that $\mathrm{cd}(P)=$ $\left\{1, p, p^{2}\right\}$ and $\operatorname{cd}(Q)=\{1, q\}$. Then, $P \times Q$ is not an SDP-group. In fact, let $P_{1} \in \max P$ such that $\chi \in \operatorname{Irr}\left(P_{1}\right)-\operatorname{Lin}\left(P_{1}\right)$. Obviously $P_{1} \times Q$ is a subgroup of $P \times Q$. However, by Theorem 4.21 of [1], there is a nonlinear character $\chi \in \operatorname{Irr}\left(P_{1} \times Q\right)$ such that $\chi$ is not a DP-character.

Example 2. Let $P$ be nonabelian $p$-group with $\operatorname{cd}(P)=\left\{1, d_{1}, d_{2}, \ldots, d_{s}\right\}, s \geq 2$, and $p>7$. Then, $A_{5} \times P$ is not an SDP-group. In fact, $A_{5}$ and $P$ are DP-groups. Let $G=A_{5} \times P$; then, there are a maximal proper subgroup $P_{1}$ of $P$ and an irreducible character $\chi \in \operatorname{Irr}\left(P_{1}\right)-\operatorname{Lin}\left(P_{1}\right)$, so $A_{5} \times P_{1}$ has a nonlinear character $\theta \chi$ for some $\theta \in \operatorname{Irr}\left(A_{5}\right)-$ $\operatorname{Lin}\left(A_{5}\right)$ and $\theta \chi$ is a non-DP-character.

There are simple groups whose certain subgroup is a DP-group, but itself not.

Example 3. Let $J_{1}$ be the simple sporadic group, and let $P$ be the Sylow 2-subgroup of $J_{1}$. Say $N=N_{G}(P)$, the normalizer of $P$ in $G$. Then, $2^{\wedge} 3: 7: 3$ is a DP-group since $\operatorname{cd}(N)=$ $\{1,3,7\}$ by [7].

Example 4. Let $M_{11}$ be the Mathieu group of degree 11. By pp.18 in [6], $M_{11}$ has a maximal subgroup $\mathrm{H}$ isomorphic to 2: $S_{4}$. By [7], $\operatorname{cd}\left(2: S_{4}\right)=\{1,2,3,4\}$ and so $H$ is a DP-group.

We need some subgroup structure of finite simple groups to shorten the proof of Theorem 3.

Lemma 2. Let $q$ be a prime power.

(1) Let $n \geq 8$. Then, $A_{n}$ has a subgroup $A_{n-1}$.

(2) Let $n \geq 4$ and $\varepsilon= \pm$. Then, $P S L_{n}^{\varepsilon}(q)$ has a subgroup isomorphic to $S L_{n-1}^{ \pm}(q)$ or $P S L_{n-1}^{ \pm}(q)$, and $S L_{n}^{\varepsilon}(q)$ has a subgroup of the form $S L_{n-1}^{\varepsilon}(q)$.

(3) Let $n \geq 2$. Then, $P S p_{2 n}(q)$ has a subgroup $P S p_{2(n-1)}(q)$.

(4) Let $n \geq 3$, and $q$ odd. Then, $\Omega_{2 n+1}(q)$ contains $a$ subgroup $\Omega_{2 n-1}(q)$.

(5) Let $n \geq 4, \varepsilon= \pm$. Then, $P \Omega_{2 n}^{\varepsilon}(q)$ has a subgroup $P \Omega_{2 n-2}^{\varepsilon}(q)$ with q odd or $P S p_{2 n-2}(q)$ with q even.

Let $P S L_{n}^{+}(q)=P S L_{n}(q)$ and $P S L_{n}^{-}(q)=P S U_{n}(q)$. Let $S L_{n}^{+}(q)=S L_{n}(q)$ and $S L_{n}^{-}(q)=S U_{n}(q)$. Let $\varepsilon= \pm$.

Proof

(1) By Appendix in [8], $S_{n}$, the symmetric group of degree $n$, has a subgroup $S_{n-k} \times S_{k}$ with $1 \leq k<n / 2$. Take $k=1, S_{n-1} \leq S_{n}$, and so $A_{n}$ has a subgroup $A_{n-1}$ as $\left|S_{n}: A_{n}\right|=2$.

(2) By Proposition 4.1.4 of [9], $\operatorname{PSL}_{n}^{ \pm}(q)$ has a maximal subgroup $H$ isomorphic to

$\left[\frac{a_{m, n-m}^{\varepsilon}}{(q-\varepsilon, n)}\right] \cdot\left(\operatorname{PSL}_{m}^{\varepsilon}(q) \times \operatorname{PSL}_{n-m}^{\varepsilon}(q)\right) \cdot\left[b_{m, n-m}^{\varepsilon}\right]$.

Take $m=1$, and $\operatorname{PSL}_{n}^{\varepsilon}(q)$ has a subgroup isomorphic to $\mathrm{SL}_{n-1}^{\varepsilon}(q)$ or $\operatorname{PSL}_{n-1}^{\varepsilon}(q)$.

We know that $\mathrm{GL}_{n}^{\varepsilon}(q)$ has a subgroup $\mathrm{GL}_{n-1}^{\varepsilon}(q)$, and $\mathrm{SL}_{n}^{\varepsilon}(q) \unlhd \mathrm{GL}_{n}^{\varepsilon}(q)$. Note that $\left(\mathrm{GL}_{n}^{\varepsilon}(q) / \mathrm{SL}_{n}^{\varepsilon}(q)\right)$ is isomorphic to a cyclic group of order $q-1, \operatorname{so~SL}_{n}^{\varepsilon}(q)$ has a subgroup isomorphic to $\mathrm{SL}_{n-1}^{\varepsilon}(q)$.

(3) Note that $\operatorname{Sp}_{2 n}(q)=2 \cdot \operatorname{PSp}_{2 n}(q)$ for odd $q$ and $\operatorname{Sp}_{2 n}(q)=\operatorname{PSp}_{2 n}(q)$ for even $q$. Then, by Table 3.5.C of [9], $\operatorname{PSp}_{2 n}(q)$ has a subgroup $\operatorname{PSp}_{2 n-2}(q)$.

(4) By Table 3.5.D of [9], $\Omega_{2 n+1}(q)$ has a subgroup with form $\Omega_{2 m+1}(q) \perp \Omega_{2(n-m)+1}^{\varepsilon}(q)$ with $1 \leq m<n, \varepsilon= \pm$. Taking $m=1, \Omega_{2 n+1}(q)$ has a subgroup $\Omega_{2 n-1}(q)$. 
(5) By Table 3.5.E of [9], $\Omega_{2 \eta}^{+}(q)$ has a subgroup of the form $P \Omega_{2 m}^{\varepsilon}(q) \perp P \Omega_{2(n-m)}^{\varepsilon}(q)$ or $\Omega_{2 m+1} \perp P \Omega_{2(n-m)}^{\varepsilon}$ (q), with $1 \leq m<n, \varepsilon \in\{+,-, \circ\}, q$ odd if $m$ odd, or the form $\operatorname{Sp}_{2 n-2}(q)$ with $q$ even.

Taking $m=1$ and $\left|\Omega_{2 n}^{+}(q): P \Omega_{2 n}^{+}(q)\right|=\operatorname{gcd}(4, q-1)$, $W_{n}$ has a subgroup $P \Omega_{2(n-1)}^{+}(q)$ or $\operatorname{PSp}_{2 n-2}(q)$.

By Table 3.5.F of [9], we also have that $\Omega_{2 n}^{-}$has a subgroup of the form either or $\Omega_{2(n-1)}^{-}(q)$ for $q$ odd or $\operatorname{Sp}_{2 n-2}(q)$ with $q$ even.

Taking $m=1$ and $\left|\Omega_{2 n}^{-}(q): P \Omega_{2 n}^{-}(q)\right|=\operatorname{gcd}(4, q+1)$, $W_{n}$ has $P \Omega_{2(n-1)}^{-}(q)$ for odd $q$ or $\operatorname{PSp}_{2(n-1)}(q)$ for even $q$.

The following three lemmas are important to give the structure of the simple groups as Theorem 3.

Lemma 3 (see [10]). The only solution of the diophantine equation $p^{m}-q^{n}=1$ with $p$ and $q$ prime and $m, n>1$ is $3^{2}-2^{3}=1$.

Lemma 4 (see $[10,11])$. With the exceptions of the relations $239^{2}-2 \cdot 13^{4}=-1$ and $3^{5}-2 \cdot 11^{2}=1$, every solution of the equation $p^{m}-2 q^{n}= \pm 1$ with $p, q$ prime, $m, n>1$, has exponents $m=n=2$, i.e., it comes from a unit $p-q \cdot 2^{(1 / 2)}$ of the quadratic field $\mathbb{Q}\left(2^{(1 / 2)}\right)$ for which the coefficients $p$ and $q$ are primes.

Lemma 5 (Zsigmondy theorem, see [12]). Let $p$ be a prime and let $n$ be a positive integer. Then, one of the following holds:

(i) There is a primitive prime $p^{\prime}$ for $p^{n}-1$, that is, $p^{\prime} \mid\left(p^{n}-1\right)$, but $p^{\prime}+\left(p^{m}-1\right)$, for every $1 \leq m<n$

(ii) $p=2, n=1$ or 6

(iii) $p$ is a Mersenne prime and $n=2$

We say that a group $A$ acts Frobeniusly on a group $B$ if $[B] A$ is a Frobenius group with kernel $B$ and complement $A$. $A$ group $G$ is a 2-Frobenius group if there is a normal series $N \triangleleft M \triangleleft G$ such that $G / N$ and $M$ are Frobenius groups with $M / N, N$ as their Frobenius kernels, respectively.

Lemma 6 (see Theorems 13.3 and 13.8 of [13]). Let $G=$ $K$ : $H$ be a Frobenius group with kernel $K$ and complement $H$. Then,

(i) $|H| \mid(|K|-1)$.

(ii) Any subgroup of $H$ of order $p^{2}$ or pq is cyclic, where $p$ and $q$ are primes.

(iii) If $|H|$ is even, $K$ is abelian.

(iv) In any case, $K$ is nilpotent.

(v) Assume that $K$ has $h(K)$ conjugacy classes, $H$ has $h(H)$ conjugacy classes, and $G$ has characters with forms:

(a) $h(H)$ is irreducible characters $\chi_{1}, \ldots, \chi_{h(H)}$ with $K$ in their kernel; if $\mu_{1}, \ldots, \mu_{h(H)}$ are the irreducible characters of $H$, then these satisfy $\chi_{i}(h k)=\mu_{i}(h)$, for all $h \in H$ and $k \in K$.

(b) Whenever $\tau \neq 1_{K}$ is an irreducible character of $K$, then $\tau^{G}$ is an irreducible character of $G$. This gives
$[h(K)-1] /|H|$ irreducible characters of $G$ with $K$ not in their kernel. Such $\tau^{G}$ satisfy

$$
\left.\tau^{G}\right|_{H}=\tau(1) \rho_{H}
$$

where $\rho_{H}$ is the regular character of $H$.

The following result is due to White [14].

Lemma 7 (see Theorem A of [14]). Let $S=P S L_{2}(q)$, with $q=p^{f}>3$, for some prime $p, A=A u t(S)$, and let $S \leq H \leq A$. Given $G=P G L_{2}(q)$ if $\bar{\delta} \in H, G=S$ if $\bar{\delta} \notin H$ and set $|H: G|=$ $d=2^{a} m$ for $m$ odd. If $p$ is odd, set $\varepsilon=(-1)^{(q-1) / 2}$. The set of irreducible character degrees of $H$ is

$$
\operatorname{cd}(H)=\left\{1, q, \frac{(q+\varepsilon)}{2}\right\} \cup\left\{(q-1) 2^{a} i: i \mid m\right\} \cup\{(q+1) j: j \mid d\},
$$

with the following exceptions:

(i) If $p$ is odd with $H \neq S\langle\varphi\rangle$ or if $p=2$, then $(q+\varepsilon) / 2$ is not a degree of $H$

(ii) If $f$ is odd, $p=3$, and $H=S\langle\varphi\rangle$, then $i \neq 1$

(iii) If $f$ is odd, $p=3$, and $H=A$, then $j \neq 1$

(iv) If $f$ is odd, $p=2,3$ or 5 , and $H=S\langle\varphi\rangle$, then $j \neq 1$

(v) If $p \equiv 2(\bmod 4), p=2$ or 3 , and $H=S\langle\varphi\rangle$, then $j \neq 2$

Theorem 3. Let $G$ be a nonabelian simple SDP-group. Then, $G$ is isomorphic to one of the groups:

(1) $\operatorname{PSL}_{2}(9), \operatorname{PSL}_{2}\left(3^{5}\right)$, and $P S L_{2}(q)$, where $q=2 r^{m}+$ $1 \geq 5$ is a prime with $r$ prime or $q=2^{m}$ and $2^{m}-1 \geq 3$ is a prime

(2) $\mathrm{PSL}_{3}(3)$

Proof. It is well-known that a nonabelian simple group $G$ is isomorphic to a sporadic simple group, a simple group of exceptional Lie type, an alternating group, or a simple group of classical Lie type. Thus, in the following, we consider the simple groups case by case.

Claim 1: if $G$ is isomorphic to $A_{n}$ with $n \geq 5$, then $G$ is isomorphic to $A_{5}$ or $A_{6}$.

If $n=7$, then $A_{7}$ has a subgroup $L_{2}$ (7) by (pp.10 in [6]) and $2.3 \in \mathrm{cd}\left(\mathrm{PSL}_{2}(7)\right)$, a contradiction. It follows that $A_{7}$ is not an SDP-group and also not a DP-group.

Let $n \geq 8$. We know from Appendix in [8] that the symmetric group $S_{n}$ of degree $n$ contains a subgroup $S_{n-1}$, so $A_{n-1}<A_{n}$. It follows from Lemma 2 that there exists a subgroup series:

$$
A_{7}<A_{8}<\cdots<A_{n-1}<A_{n},
$$

so $A_{n}$ with $n \geq 8$ is not an SDP-group.

If $n=6$, then by pp.4 in [6], $\max A_{6}=\left\{A_{5}, 3^{2}: 4, S_{4}\right\}$. We can see that $\operatorname{cd}\left(A_{5}\right)=\{1,3,4,5\}, \operatorname{cd}\left(3^{2}: 4\right)=\{1,4\}$, and $\operatorname{cd}\left(S_{4}\right)=\{1,2,3\}$, so $G \cong A_{6}$ is an SDP-group by Lemma 1 . If $n=5$, then by Theorem 1 and Lemma $1, A_{5}$ is an SDP-group. 
It follows that $G$ is isomorphic to $A_{5}$ or $A_{6}$.

Claim 2: a sporadic simple group or a Tits group is not an SDP-group.

Let $G$ be a sporadic simple group or a Tits group and let $H$ be a proper subgroup of $G$; then, we can get Table 1 by [6] and Theorem 4.21 of [1]. For each $\chi_{i} \in \operatorname{Irr}(H), \chi_{i}$ is not a DP-character. It follows that a sporadic simple group or a Tits group is a non-SDP-group.

Claim 3: if $G$ is isomorphic to a simple group of classical Lie type, then $G$ is isomorphic to $\operatorname{PSL}_{2}(9), \operatorname{PSL}_{2}\left(3^{5}\right)$, $\operatorname{PSL}_{2}(q)$, where $q=2 r^{m}+1 \geq 5$ is a prime with $r$ a prime or $q=2^{m}$ and $2^{m}-1 \geq 3$ is a prime, and $\mathrm{PSL}_{3}(3)$.

\section{1. $\operatorname{PSL}_{n}(q), n \geq 2$}

2.1.1. Let $n=2$. If $q=4$ or 8 , then $\mathrm{PSL}_{2}(4)$ and $\mathrm{PSL}_{2}(8)$ are DP-groups by Theorem 1, so Lemma 1 (3) implies that $\mathrm{PSL}_{2}$ (4) and $\mathrm{PSL}_{2}(8)$ are SDP-groups. It follows that $G$ is isomorphic to $\mathrm{PSL}_{2}(4)$ or $\mathrm{PSL}_{2}$ (8). Thus, $q \geq 9$ or $q=7$. If $q=7$, then by pp.3 in [6], all maximal subgroups of $\mathrm{PSL}_{2}(7) \cong \mathrm{PSL}_{3}(2)$ are of the forms $S_{4}$ and 7: 3 . Note that $\operatorname{cd}\left(S_{4}\right)=\{1,2,3\}$ and $\operatorname{cd}(7: 3)=\{1,3\}$, so $\mathrm{PSL}_{2}(7)$ is an SDP-group. Now, $q \geq 9$. If $q=9$, then by pp.5 in [6], $\max L_{2}(9)=\left\{A_{5}, 3^{2}: 4, S_{4}\right\}$; hence, $\mathrm{PSL}_{2}(9)$ is an SDP-group since $A_{5}$ is a DP-group, $\operatorname{cd}\left(3^{2}: 4\right)=\{1,4\}$ and $\operatorname{cd}\left(S_{4}\right)=$ $\{1,2,3\}$. Therefore, $G$ is isomorphic to $\mathrm{PSL}_{2}(7)$ or $\mathrm{PSL}_{2}(9)$.

By Table 2, $\operatorname{PSL}_{2}(q)$ has a subgroup of the form $E_{q}: Z_{(q-1) / 2}$. Note from Lemma 6 that $\operatorname{cd}\left(E_{q}: Z_{(q-1) / 2}\right)=\{1,(q-1) / 2\}$, so by hypothesis, $(q-1) / 2$ is a prime power or a prime. Two cases now are considered as follows: $q>9$ odd and $q \geq 16$ even.

Case 1: $q>9$ odd.

Let $q=p^{m}$ with $m \geq 2$. We first assume that $(q-1) / 2$ is a prime. If $m \geq 2$ is even, then $(p-1)(p+1)$ divides $\left(p^{m}-1\right)$. Note that $(p-1)_{2}(p+1)_{2}=2^{k}$, for some $k \geq 3$, so assumption shows $(q-1) / 2=2^{s}$ for some $s \geq 3$. Now, Lemma 3 shows that $((q-1) / 2)=2^{s}$ has no solution in $\mathbb{N}$. If $m \geq 3$ is odd, then $((q-1) / 2)=((p-1) / 2) \cdot\left(p^{m-1}+p^{m-2}+\cdots+1\right)$. It is easy to see that $p^{m-1}+p^{m-2}+\cdots+1$ is odd, so $(q-$ $1) / 2$ cannot be a prime. Assume now that $(q-1) / 2$ is a prime power and say $q-1=2 r^{n}$ for some integer $n \geq 0$ and a prime $r$.

(i) If $n=0$, then $q=3$, but $\operatorname{PSL}_{2}$ (3) is solvable, a contradiction.

(ii) If $n \geq 1$ and $r=2$, then by Lemmas 3-5, $q=5,9>9$, or $q=2^{n+1}+1$ is a prime. It follows that $G$ is isomorphic to $\operatorname{PSL}_{2}(q)$ with $q=2^{n+1}+$ $1 \geq 17$ a prime.

(iii) If $n \geq 2$ and $r \geq 3$, then by Lemma $4, q=3^{5}$, $r=11$, and $n=2$ or $q-1=p^{2}-1=(p+$ 1) $(p-1)=2 r^{2}$ (rule out this case since 8 divides $\left.(p+1)_{2}(p-1)_{2}\right)$. So, we have $G \cong \operatorname{PSL}_{2}\left(3^{5}\right)$.
TABLE 1: Simple sporadic groups.

\begin{tabular}{lccc}
\hline$G$ & $H$ & Character & Degree \\
\hline$M_{11}$ & $\mathrm{PSL}_{2}(11)$ & $\chi_{4}$ & 2.5 \\
$J_{1}$ & $\mathrm{PSL}_{2}(11)$ & $\chi_{4}$ & 2.5 \\
$J_{2}$ & $A_{4} \times A_{5}$ & $\chi_{20}$ & 3.5 \\
$\mathrm{HS}$ & $M_{11}$ & $\chi_{2}$ & 2.5 \\
$M_{24}$ & $M_{23}$ & $\chi_{2}$ & 2.11 \\
$\mathrm{He}$ & $\mathrm{PSL}_{3}(2)$ & $\chi_{4}$ & 2.3 \\
$\mathrm{Suz}$ & $A_{7}$ & $\chi_{2}$ & 2.3 \\
$\mathrm{Co}_{3}$ & $A_{4} \times S_{5}$ & $\chi_{3}$ & 2.3 \\
$\mathrm{Fi}_{22}$ & $S_{10}$ & $\chi_{5}$ & 5.7 \\
$\mathrm{Ly}$ & $G_{2}(5)$ & $\chi_{2}$ & $2^{2} \cdot 31$ \\
$\mathrm{Fi}_{23}$ & $S_{12}$ & $\chi_{7}$ & 5.11 \\
$J_{4}$ & $2^{11}: M_{24}$ & $\chi_{3}$ & $3^{2} \cdot 5$ \\
$B$ & $S_{4} \times{ }^{2} F_{4}(2)$ & $\chi_{3}$ & $2^{2} \cdot 13$ \\
${ }^{2} F_{4}(2)^{\prime}$ & $M_{11}$ & $\chi_{2}$ & 2.5 \\
$M_{12}$ & $M_{11}$ & $\chi_{2}$ & 2.5 \\
$M_{22}$ & $\mathrm{PSL}_{2}(11)$ & $\chi_{4}$ & 2.5 \\
$M_{23}$ & $A_{7}$ & $\chi_{2}$ & 2.3 \\
$J_{3}$ & $\mathrm{PSL}_{2}(19)$ & $\chi_{4}$ & $2 \cdot 3^{2}$ \\
$\mathrm{McL}_{\mathrm{Ru}}$ & $M_{22}$ & $\chi_{2}$ & 3.7 \\
$\mathrm{RN}$ & $A_{8}$ & $\chi_{3}$ & 2.7 \\
$\mathrm{Co}{ }_{2}$ & $A_{7}$ & $\chi_{2}$ & 2.3 \\
$\mathrm{HN}$ & $M_{23}$ & $\chi_{2}$ & 2.11 \\
$\mathrm{Th}$ & $A_{12}$ & $\chi_{4}$ & 5.11 \\
$\mathrm{Co}$ & $\mathrm{PSL}_{2}(19): 2$ & $\chi_{3}$ & $2 \cdot 3^{2}$ \\
$\mathrm{Fi}_{24}{ }^{\prime}$ & $A_{9} \times S_{3}$ & $\chi_{7}$ & 3.7 \\
$M$ & $\mathrm{He}_{2}$ & $\chi_{3}$ & 2.3 .17 \\
\hline & $2^{5}: S_{6}$ & $\chi_{17}$ & 2.5 \\
\hline & & &
\end{tabular}

TABLe 2: $\operatorname{PSL}_{2}(q), q \geq 5$ (chap II, Theorem 8.27 of [15]).

\begin{tabular}{lcc}
\hline & $\max L_{2}(q)$ & Condition \\
\hline $\mathscr{C}_{1}$ & $E_{q}: Z_{(q-1) / k}$ & $k=\operatorname{gcd}(q-1,2)$ \\
$\mathscr{C}_{2}$ & $D_{2(q-1) / k}$ & $q \notin\{5,7,9,11\}$ \\
$C_{3}$ & $D_{2(q-1) / k}$ & $q \notin\{7,9\}$ \\
$\mathscr{C}_{5}$ & $\mathrm{PSL}_{2}\left(q_{0}\right) \cdot(k, b)$ & $q=q_{0}^{b}, b$ a prime, $q_{0} \neq 2$ \\
$\mathscr{C}_{6}$ & $S_{4}$ & $q=p \equiv \pm 1(\bmod 8)$ \\
& $A_{4}$ & $q=p \equiv 3,5,13,27,37(\bmod 40)$ \\
$\mathcal{S}$ & $A_{5}$ & $q \equiv \pm 1(\bmod 10), F_{q}=F_{p}[\sqrt{5}]$ \\
\hline
\end{tabular}

Let $q=p$ be a prime. Then, the fact that $(q-1) / 2$ is a prime or a prime power implies that $q=p=1+2 r^{m}$, where $m \geq 1$ is an integer and $r$ is a prime, so $G \cong$ $\operatorname{PSL}_{2}(q)$ with $q=1+2 r^{m}$, for some integer $m \geq 1$ and $r$ a prime.

Case 2: $q \geq 2^{4}$ even.

Now, Table 2 implies that $\operatorname{PSL}_{2}(q)$ has a maximal subgroup $E_{q}: Z_{q-1}$. By Lemma 6, $\operatorname{cd}\left(E_{q}: Z_{q-1}\right)=\{1, q-1\}$. By assumption, $q-1$ is a prime or a prime power. If $q-1$ is a prime and say $q=2^{m} \geq 2^{4}$, then $G \cong \operatorname{PSL}_{2}(q)$ with $q=2^{m} \geq 2^{4}, q-1$ a prime. If $q-1$ is a prime power, then by Lemma 3 , it is impossible.

2.1.2. Let $n=3$. If $q=2$, then since $\mathrm{PSL}_{3}(2) \cong \mathrm{PSL}_{2}(7)$, one has that $\mathrm{PSL}_{3}(2)$ is an SDP-group. If $q=3$, then by [6], $\mathrm{PSL}_{3}(3)$ has maximal subgroups with the forms: $3^{2}: 2 S 4$, 
13: 3 , and $S 4$, so by GAP, one obtains that $\operatorname{cd}\left(3^{2}: 2 S 4\right)=\operatorname{cd}\left(M_{9}: S_{3}\right)=\{1,2,3,4,8,16\}$, so $G \cong \mathrm{PSL}_{3}$ (3). If $q=4$, then by pp.23 in [6], $\mathrm{PSL}_{3}(4)$ has a maximal subgroup $\mathrm{PSL}_{2}(7)$, but $2.3 \in \mathrm{cd}\left(\mathrm{PSL}_{2}(7)\right)$, a contradiction to the hypothesis. Now, we assume that $q \geq 5$. By Table 3, $\operatorname{PSL}_{3}(q)$ has a subgroup $E_{q^{2}}:(1 / k) \mathrm{GL}_{2}(q)>\mathrm{PGL}_{2}(q)$. Note that $\mathrm{cd}\left(\mathrm{PGL}_{2}(q)\right)=\{1, q, q-1, q+1\}$. By assumption, $q-1$ and $q+1$ are prime powers. Note that $q-1, q$, and $q+1$ are consecutive integers, so one of the numbers $q-1, q, q+1$ is divisible by 3 .

Let $q=p^{m}$ be odd with $m \geq 2$. Then, $q=3^{n}$ for some integer $n$. Since $q \geq 5$, one has that $n \geq 2$ and that $q-1$ and $q+1$ are divisible by 2 . It follows that $q=3 ¥ 5$, a contradiction.

Let $q=p^{m}$ be even with $m \geq 3$. Then, 3 divides $q-1$ or $q+1$. If $q-1=3$ or $q-1=3^{s}$, for some $s \geq 2$, then, by Lemma $3, q=4 \nsupseteq 5$ a contradiction. If $q+1=3$ or $q+1=3^{s}$, for some $s \geq 2$, then $q=2$ or $q=8$ by Lemma 3. If $G \cong \mathrm{PSL}_{3}(8)$, then, by pp.74 in [6], $\mathrm{PSL}_{3}(8)$ has a subgroup
TABLE 3: $\operatorname{PSL}_{3}(q)$ (see page 191 of $\left.[16]\right)$

\begin{tabular}{ccc}
\hline & $\max L_{3}(q)$ & Condition \\
\hline $\mathscr{C}_{1}$ & $E_{q^{2}}:(1 / \mathrm{k}) \mathrm{GL}_{2}(q)$ & $k=\operatorname{gcd}(3, q-1)$ \\
\hline $\mathscr{C}_{2}$ & $Z_{(q-1) / k}^{2} \cdot S_{3}$ & $q \geq 5$ \\
\hline$C_{3}$ & $Z_{\left(q^{2}+q+1\right) / k} \cdot 3$ & $q \neq 4$ \\
\hline $\mathscr{C}_{5}$ & $\mathrm{PSL}_{3}\left(q_{0}\right) \cdot(\delta, b)$ & $q=q_{0}^{b}, b$ prime \\
\hline $\mathscr{C}_{6}$ & $3^{2} \cdot \mathrm{SL}_{2}(3)$ & $q=p \equiv 1(\bmod 9)$ \\
& $3^{2} \cdot Q_{8}$ & $q=p \equiv 4,7(\bmod 9)$ \\
$\mathscr{C}_{8}$ & $\mathrm{SO}_{3}(q)$ & $q$ odd \\
& $\mathrm{PSU}_{3}\left(q_{0}\right)$ & $q=q_{0}^{2}$ \\
\hline \multirow{S}{S}{} & $A_{6}$ & $p \equiv 1,2,4,7,8,13,(\bmod 15)$, \\
& $\mathrm{PSL}_{2}(7)$ & $F_{q}=F_{p}[\sqrt{5}, \sqrt{-3}]$ \\
& & $2<q=p \equiv 1,2,4(\bmod 7)$ \\
\hline
\end{tabular}

$\mathrm{PSL}_{2}(7)$ and $2.3 \in \mathrm{cd}\left(\mathrm{PSL}_{2}(7)\right)$, a contradiction to the hypothesis.

2.1.3. Let $n \geq 4$. We know from [17] that

$$
\operatorname{cd}\left(\operatorname{PSL}_{3}(q)\right)=\left\{1, q(q+1), q^{2}+q+1,(q+1)(q-1)^{2},(q-1)\left(q^{2}+q+1\right), q^{3}, q\left(q^{2}+q+1\right),(q+1)\left(q^{2}+q+1\right)\right\}
$$

so $\operatorname{PSL}_{3}(q)$ is not a DP-group.

Now, Lemma 2 shows that there exists a subgroup series:

$$
\begin{gathered}
\operatorname{PSL}_{3}(q)<\operatorname{PSL}_{n}(q) \\
\text { or } \mathrm{SL}_{3}(q)<\operatorname{PSL}_{n}(q),
\end{gathered}
$$

so $\operatorname{PSL}_{n}(q)$ for $n \geq 4$ is a non-SDP-group.

\section{2. $\operatorname{PSU}_{n}(q), n \geq 3$}

2.2.1. Let $n=3$. If $q=2$, then $\operatorname{PSU}_{3}(2) \cong 3^{2}: Q 8$ is solvable, so $q \geq 3$. If $q=3$, then $\mathrm{PSU}_{3}(3)$ has a subgroup $\mathrm{PSL}_{2}$ (7) by pp.14 in [6], so $2.3 \in \mathrm{cd}\left(\mathrm{PSL}_{2}(7)\right)$, a contradiction. Hence, $q \geq 4$, so by pp.200 in [16], $\operatorname{PSU}_{3}(q)$ has a subgroup $\mathrm{GU}_{2}(q)$. Notice that $\operatorname{PSU}_{2}(q) \cong \mathrm{PSL}_{2}(q)$ implies $\mathrm{GU}_{2}(q) \cong \mathrm{GL}_{2}(q)$. Thus, by Table 2 in [18], $\mathrm{cd}\left(\mathrm{GU}_{2}(q)\right)=\{1, q-1, q, q+1\}$. By hypothesis, $|\pi(q-1)|=1=|\pi(q+1)|$, so $q=4$ or $q=8$ by Lemmas 3 and 5. If $q=4$, then $\operatorname{PSU}_{3}(4)$ has a maximal subgroup $2 \wedge(2+4): 15$, so by [7], $\operatorname{cd}(2 \wedge(2+4): 15)$ $=\left\{1,2^{2} .3,3.5\right\}$, a contradiction. If $q=8$, then, by pp.66 in [6], $\operatorname{PSU}_{3}(8)$ has a subgroup $2 \wedge(3+6): 21$, whence by [7], $\operatorname{cd}(2 \wedge(3+6): 21)=\left\{1,3.7,2^{3} \cdot 7\right\}$, a contradiction to the assumption.

2.2.2. Let $n \geq 4$. We see from [17] that

$$
\operatorname{cd}\left(\operatorname{PSU}_{3}(q)\right)=\left\{1, q(q-1), q^{2}-q+1,(q-1)\left(q^{2}-q+1\right), q\left(q^{2}-q+1\right), q^{3},(q+1)\left(q^{2}-q+1\right),(q-1)(q+1)^{2}\right\}
$$

so $\operatorname{PSU}_{3}(q)$ is not a DP-group.

By Lemma 2, one has

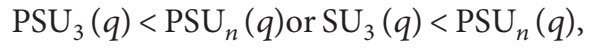

so $\operatorname{PSU}_{n}(q)$ for $n \geq 4$ is not a SDP-group.

2.3. $P S p_{2 n}(q)$ with $n \geq 2, \Omega_{2 n+1}(q)$ with $n \geq 3$, q Odd, $P \Omega_{2 n}^{\varepsilon}(q)$ with $n \geq 4$, or a Simple Group of Exceptional Lie Type.

First, we consider the simple groups of exceptional Lie type, where $N\left(A_{i}\right):{ }^{2} G_{2}(q)$ has abelian Hall subgroups $A_{2}$ and $A_{3}$ of order $q+1-3^{m+1}$ and $q+1+3^{m+1}$, respectively; $N\left(A_{i}\right)$ is a Frobenius group with kernel $A_{i}, i=2,3$, and a cyclic noninvariant factor of order 6 . $A \circ B$ denotes the central product of $A$ and $B$.

Consider ${ }^{2} G_{2}(q)$; then, by Table $4, G$ has a Frobenius subgroup $N\left(A_{i}\right)$ with cyclic complement of order 6 and abelian kernel $A_{i}$ of order $q \pm \sqrt{3 q}+1$, i.e., $N\left(A_{i}\right) / A_{i}$ is isomorphic to a cyclic group of order 6. It follows that $N\left(A_{i}\right) / A_{i}$ is abelian, so $\left|N\left(A_{i}\right) / A_{i}\right| \in \operatorname{cd}\left(N\left(A_{i}\right)\right)$. This means that there is a proper subgroup $H$ of $G$ and a character $\chi \in \operatorname{Irr}(H)$ such that $\chi(1)$ is divisible by 6 , so $\chi$ is not a DP-character. It follows that ${ }^{2} G_{2}(q)$ is a nonSDP-group. 
TABLE 4: A proper maximal subgroup $H$ of $G$.

\begin{tabular}{lccc}
\hline$G$ & $H$ & Condition & References \\
\hline${ }^{2} G_{2}(q)$ & $N\left(A_{i}\right)$ & $q=3^{2 m+1} \geq 27$ & {$[19]$} \\
\hline$G_{2}(q)$ & $\mathrm{SL}_{3}(q) \cdot 2$ & $3 \mid q$ & $($ Table 1 in \\
& $\mathrm{SL}_{3}(q) \cdot 2$ & $3+q$ & $[20])$ \\
\hline${ }^{3} D_{4}(q)$ & $G_{2}(q)$ & $q$ square & $\begin{array}{c}\text { Table } 1 \text { in } \\
[20])\end{array}$ \\
\hline${ }^{3} D_{4}(\sqrt{q})$ & & $\begin{array}{c}\text { (Table } 1 \text { in } \\
[20])\end{array}$ \\
\hline$F_{4}(q)$ & ${ }^{3} D_{4}(q) \cdot S_{3}$ & $\varepsilon= \pm 1$ & $\begin{array}{c}\text { (Table } 1 \text { in } \\
[20])\end{array}$ \\
\hline$E_{7}(q)$ & $\left(\mathrm{SL}_{2}(q) \circ P \Omega_{12}^{+}(q)\right) \cdot d$ & $|Z(L \cap M)|=d$ & $\begin{array}{c}(\text { Table } 1 \text { in } \\
[20])\end{array}$ \\
\hline$E_{8}(q)$ & $\left(\mathrm{SL}_{2}(q) \circ E_{7}(q)\right) \cdot d$ & $|Z(L \cap M)|=d$ & $\begin{array}{c}\text { (Table 1 in } \\
[20])\end{array}$ \\
\hline
\end{tabular}

Now, we do with other cases. By Table 4, we obtain subgroup series

$$
E_{8}(q)>E_{7}(q)>P \Omega_{12}(q)
$$

and either

$E_{6}^{\varepsilon}(q)>F_{4}(q)>{ }^{3} D_{4}(q)>G_{2}(q)>\operatorname{SL}(3, q) \cdot 2>\mathrm{SL}_{3}(q), \quad$ for $3 \mid q$,

or

$E_{6}^{\varepsilon}(q)>F_{4}(q)>{ }^{3} D_{4}(q)>G_{2}(q)>\mathrm{SU}(3, q) \cdot 2>\mathrm{SU}_{3}(q), \quad$ for $3+q$.

Note that $P \Omega_{12}^{+}(q)>P \Omega_{4}^{+}(q)$ and that $\operatorname{PSL}_{3}(q) \cong\left(\mathrm{SL}_{3}(q) / Z\left(\mathrm{SL}_{3}(q)\right)\right)$ and $\operatorname{PSU}_{3}\left(q^{2}\right) \cong$ $\left(\mathrm{SU}_{3}\left(q^{2}\right) / Z\left(\mathrm{SU}_{3}\left(q^{2}\right)\right)\right)$ are non-DP-groups, so $E_{8}(q)$, $E_{7}(q), E_{6}^{\varepsilon}(q), F_{4}(q),{ }^{3} D_{4}(q)$, and $G_{2}(q)$ are nonSDP-groups.

Now, consider ${ }^{2} B_{2}(q)$ with $q=2^{2 m+1} \geq 8$; then, by Theorem 9 of $[21],{ }^{2} B_{2}(q)$ has a subgroup $E_{q}^{1+1}: Z_{q-1}$, and $d(q-1) \in \operatorname{cd}\left(E_{q}^{1+1}: Z_{q-1}\right)$, where $q=2^{2 m+1} \geq 8$ and $\chi(1)=d \neq 1$, for $\chi \in \operatorname{Irr}\left(E_{q}^{1+1}\right)$. It follows that $E_{q}^{1+1}: Z_{q-1}$ is not a DP-group, so ${ }^{2} B_{2}(q)$ is a nonSDP-group.

Second, we consider the groups: $\operatorname{PSp}_{2 n}(q)$ with $n \geq 2$, $\Omega_{2 n+1}(q)$ with $n \geq 3, q$ odd, $P \Omega_{2 n}^{\varepsilon}(q)$ with $n \geq 4$.

Notice that $P \Omega_{4}^{+}(q)=\operatorname{PSL}_{2}(q) \times \operatorname{PSL}_{2}(q)$, and $P \Omega_{4}^{-}(q)=\operatorname{PSL}_{2}\left(q^{2}\right)$ with $q>2, G_{2}(q)$, and $\Omega_{7}(q)$ are nonDP-groups. It follows from Table 5 and Lemma 2 that the groups $\operatorname{PSp}_{2 n}(q)$ with $n \geq 2, \Omega_{2 n+1}(q)$ with $n \geq 3, q$ is odd, and $P \Omega_{2 n}^{\varepsilon}(q)$ with $n \geq 4$ are non-SDP-group. So, we need to consider $P \Omega_{8}^{-}(2)$. We know from pp. 89 in [6] that $P \Omega_{8}^{-}(2)$ has a $\mathrm{PSL}_{2}(7)$ as a subgroup. As $2.3 \in \mathrm{cd}\left(\mathrm{PSL}_{2}(7)\right), P \Omega_{8}^{-}(2)$ contains a non-DP-subgroup, so $P \Omega_{8}^{-}(2)$ is a nonSDP-group.

\section{Proof of Theorem 2}

Now, we will prove Theorem 2.
TABLE 5: $\quad \operatorname{PSp}_{2 n}(q), \quad n \geq 2, \quad \Omega_{2 n+1}(q), \quad n \geq 3, \quad q$ is odd, and $P \Omega_{2 n}^{\varepsilon}(q), n \geq 4$.

\begin{tabular}{lccc}
\hline$G$ & $H$ & Condition & References \\
\hline$P S p_{4}(q)$ & $P \Omega_{4}^{+}(q)$ & $q$ even & (pp. 209 in [16]) \\
& $P \Omega_{4}^{-}(q)$ & $q$ odd & (pp. 209 in [16]) \\
\hline$\Omega_{7}(q)$ & $G_{2}(q)$ & $q$ odd & (pp. 213 in $[16])$ \\
\hline$P \Omega_{8}^{+}(q)$ & $\Omega_{7}(q)$ & & (pp. 214 in [16]) \\
\hline$P \Omega_{8}^{-}(q)$ & $P \Omega_{4}^{-}(q)$ & & (pp. 215 in $[16])$ \\
\hline
\end{tabular}

Let $q=p^{f}$ for some prime $p$. Note that the order of outer-automorphism group of $\mathrm{PSL}_{2}(q)$ is

$$
\left|\operatorname{Out}\left(\mathrm{PSL}_{2}(q)\right)\right|=\operatorname{gcd}(2, q-1) \cdot f \text {. }
$$

\subsection{Proof of Theorem 2}

Proof. If $G$ is simple, then by Theorem 3, $G$ is isomorphic to one of the groups. $\operatorname{PSL}_{2}(q)$, where $q=2 r^{m}+1 \geq 5$, is a prime with $r$ an odd prime or $q=2^{m}$ and $2^{m}-1 \geq 3$ a prime; $\mathrm{PSL}_{2}(9) ; \mathrm{PSL}_{2}\left(3^{5}\right)$. So, if $G$ is nonsimple, then we consider the following cases: $q=2^{f}$ with $2^{f}-1$ a prime, and $q=9$, $q=3^{5}$, and $q=2 r^{m}+1 \geq 5$ is a prime with $r$ an odd prime and $\mathrm{PSL}_{3}(3)$.

Case 1: $q=2^{f}$ with $2^{f}-1$ a prime.

If $f=2$, then $\mathrm{PSL}_{2}(4) \cong \mathrm{PSL}_{2}(5) \cong A_{5}$ and $\mid$ Out $\left(A_{5}\right) \mid=2$. As $G$ is a not simple group, $G$ is possible isomorphic to $S_{5}$, where $S_{n}$ is the symmetric group of degree $n$. By pp. 2 in [6], $\max S_{5}=\left\{A_{5}, S_{4}, 5: 4,2 \times S_{3}\right\}$. Note that $\operatorname{cd}\left(A_{5}\right)=\{1,3,4,5\}, \quad \operatorname{cd}\left(S_{4}\right)=\{1,2,3\}$, $\operatorname{cd}(5: 4)=\{1,4\}$, and $\operatorname{cd}\left(S_{3}\right)=\{1,2\}$, so $S_{5}$ is an SDP-group. Thus, $G \cong S_{5}$.

If $f=3$, then, by pp. 6 in [6], 7: $6 \in \max _{\mathrm{PSL}_{2}}(8) \cdot 3$, so $\mathrm{PSL}_{2}(8) \cdot 3$ is not an SDP-group.

If $f \geq 5$, then we easily get that $f$ does divides the order of $\operatorname{PSL}_{2}\left(2^{f}\right)$, so $\operatorname{PSL}_{2}\left(2^{f}\right)$ is a maximal subgroup of $\operatorname{PSL}_{2}\left(2^{f}\right) \cdot f$. We see that $\mathrm{PSL}_{2}\left(2^{f}\right)$ for $f \geq 5$ is not a DP-group, so $\mathrm{PSL}_{2}\left(2^{f}\right) \cdot f$ is a non-SDP-group.

Case $2: q=9$.

Let $\widetilde{\mathfrak{S}}=\left\{\mathrm{PSL}_{2}(9) \cdot 2_{1}, \mathrm{PSL}_{2}(9) \cdot 2_{2}, \quad \mathrm{PSL}_{2}(9) \cdot 2_{3}\right.$, $\left.\mathrm{PSL}_{2}(9) \cdot 2^{2}\right\}$.

Now, by pp. 4 in [6], $\left|\operatorname{Out}\left(\mathrm{PSL}_{2}(9)\right)\right|=4$, so $G$ is possibly isomorphic to $A \in \mathbb{S}$. Note that $\mathrm{PSL}_{2}(9) \in \sum A$ and that $\mathrm{PSL}_{2}$ (9) is a non-DP-group, so each $A \in S$ is a non-SDP-group.

Case 3: $q=3^{5}$.

Now, $\mid$ Out $\left(\mathrm{PSL}_{2}\left(3^{5}\right)\right) \mid=2 \cdot 5$. Thus, $G$ possibly is isomorphic to $\mathrm{PGL}_{2}\left(3^{5}\right), \mathrm{PGL}_{2}\left(3^{5}\right) \cdot 5, \mathrm{PSL}_{2}\left(3^{5}\right) \cdot 5$, or $\operatorname{PSL}_{2}\left(3^{5}\right) \cdot 10$. It is easy to see that $\operatorname{PSL}_{2}\left(3^{5}\right)$ is a maximal subgroup of $\operatorname{PSL}_{2}\left(3^{5}\right) \cdot 2$. So, $\operatorname{PSL}_{2}\left(3^{5}\right) \cdot 5$ is a non-SDP-group as $\mathrm{PSL}_{2}\left(3^{5}\right)$ is not a DP-group and so are $\mathrm{PSL}_{2}\left(3^{5}\right) \cdot 10$ and $\mathrm{PGL}_{2}\left(3^{5}\right) \cdot 5$. We know that $\mathrm{PGL}_{2}\left(3^{5}\right)$ has a normal subgroup $\mathrm{PSL}_{2}\left(3^{5}\right)$ of index 2 , but $\mathrm{PSL}_{2}\left(3^{5}\right)$ is a non-DP-group. Thus, $\mathrm{PGL}_{2}\left(3^{5}\right)$ is a non-SDP-group. 
Case 4: $q=2 r^{m}+1$ is a prime with $r$ an odd prime. In this case, $\left|\operatorname{Out}\left(\mathrm{PSL}_{2}(q)\right)\right|=2$. We know that $\mathrm{PSL}_{2}(q) \unlhd \mathrm{PGL}_{2}(q)$ and that $\operatorname{PSL}_{2}(q)$ is a nonDP-group. Hence, $\mathrm{PGL}_{2}(q)$ is not an SDP-group.

Case 5: $\mathrm{PSL}_{3}(3)$.

Now, $\left|\operatorname{Out}\left(\mathrm{PSL}_{3}(3)\right)\right|=2$. From pp. 13 in [6], 13: $6 \in \max \mathrm{PSL}_{3}(3) \cdot 2$, so $6 \in \operatorname{cd}(13: 6)$ is not a prime power. Thus, $\mathrm{PSL}_{3}(3) \cdot 2$ is a non-SDP-group.

\section{Data Availability}

No data were used to support the findings of the study.

\section{Conflicts of Interest}

The authors declare that they have no conflicts of interest.

\section{Acknowledgments}

The project was supported by the NSF of China (Grant no 11871360) and Opening Project of Sichuan Province University Key Laborstory of Bridge Non-destruction Detecting and Engineering Computing (Grant no 2019QYJ02).

\section{References}

[1] I. M. Isaacs, Character Theory of Finite Groups, Dover Publications, Inc., New York, NY, USA, 1994.

[2] I. M. Isaacs and D. S. Passman, "A characterization of groups in terms of the degrees of their characters. II," Pacific Journal of Mathematics, vol. 24, pp. 467-510, 1968.

[3] D. S. Passman, "Groups whose irreducible representations have degrees dividing $p^{2}$," Pacific Journal of Mathematics, vol. 17, pp. 475-496, 1966.

[4] O. Manz, "Endliche auflösbare gruppen, deren sämtliche charaktergrade primzahlpotenzen sind," Journal of Algebra, vol. 94, no. 1, pp. 211-255, 1985.

[5] O. Manz, "Endliche nicht-auflösbare gruppen, deren sämtliche charaktergrade primzahlpotenzen sind," Journal of Algebra, vol. 96, no. 1, pp. 114-119, 1985.

[6] J. H. Conway, R. T. Curtis, S. P. Norton, R. A. Parker, and R. A. Wilson, Atlas of Finite Groups: Maximal Subgroups and Ordinary Characters For Simple Groups, Oxford University Press, Oxford, UK, 1985.

[7] T. Breuer, "The GAP character table library," 2012, http:// www.math.rwth-aachen.de/Thomas.Breuer/ctbllib.

[8] M. Aschbacher and L. Scott, "Maximal subgroups of finite groups," Journal of Algebra, vol. 92, no. 1, pp. 44-80, 1985.

[9] P. Kleidman and M. Liebeck, The Subgroup Structure of the Finite Classical Groups, Cambridge University Press, Cambridge, CA, USA, volume 129 of London Mathematical Society Lecture Note Series, 1990.

[10] A. Khosravi and B. Khosravi, "A new characterization of some alternating and symmetric groups. II," International Journal of Mathematics and Mathematical Sciences, vol. 30, no. 4, pp. 953-967, 2004.

[11] P. Crescenzo, "A Diophantine equation which arises in the theory of finite groups," Advances in Mathematics, vol. 17, no. 1, pp. 25-29, 1975.

[12] K. Zsigmondy, "Zur theorie der potenzreste," Monatshefte für Mathematik und Physik, vol. 3, no. 1, pp. 265-284, 1892.
[13] L. Dornhoff, Group Representation Theory. Part A: Ordinary Representation Theory, Marcel Dekker, Inc., New York, NY, USA, 1971.

[14] D. L. White, "Character degrees of extensions of $\mathrm{PSL}_{2}(q)$ and $\mathrm{SL}_{2}(q)$," Journal of Group Theory, vol. 16, no. 1, pp. 1-33, 2013.

[15] B. Huppert, Endliche Gruppen. I. Die Grundlehren der Mathematischen Wissenschaften, Band 134, Springer-Verlag, Berlin, Germany, 1967.

[16] P. B. Kleidman, The subgroup structure of some finite simple groups, (Ph.D. Thesis), Trinity College, Cambridge, CA, USA, 1987.

[17] F. Lübeck, "Character degrees and their multiplicities for some groups of lie type of rank <9," 2007, https://www.math. rwth-aachen.de/Frank.Luebeck/chev/DegMult/index.html? $\mathrm{LANG}=\mathrm{en}$.

[18] W. A. Simpson and J. S. Frame, "The character tables for SL (3, q), SU $\left(3, q^{2}\right)$, PSL $(3, q)$, PSU $\left(3, q^{2}\right)$," Canadian Journal of Mathematics, vol. 25, pp. 486-494, 1973.

[19] V. M. Levchuk and Y. N. Nuzhin, "The structure of ree groups," Algebra I Logika, vol. 24, no. 1, pp. 26-41, 1985.

[20] M. W. Liebeck and J. Saxl, "On the orders of maximal subgroups of the finite exceptional groups of lie type," Proceedings of the London Mathematical Society, vol. 55, no. 2, pp. 299330, 1987.

[21] M. Suzuki, "On a class of doubly transitive groups," Annals of Mathematics, vol. 79, no. 2, pp. 105-145, 1962. 